VIDEO/MULTIMEDIA ARTICLE
pISSN 2234-778x • eISSN 2234-5248
J Minim Invasive Surg 2020;23(2):103-105

\title{
Robotic Lateral Pelvic Lymph Node Dissection: Description of A Technique
}

\author{
Suhail Abdullah Alturkistani, M.D. ${ }^{1}$, Alanoud Mohammed Alghanem, M.D. ${ }^{2}$, In Kyu Lee, Ph.D. ${ }^{3}$ \\ 'Department of Surgery, King Abdulaziz University Hospital, 2Department of Surgery, King Fahad Armed Forces Hospital, Jeddah, Saudi Arabia, \\ ${ }^{3}$ Division of Colorectal Surgery, Department of Surgery, Seoul St. Mary's Hospital, The Catholic University of Korea College of Medicine, Seoul, Korea
}

Different surgical approaches have been reported in the management of lateral pelvic lymph node dissection (LPND) including open, laparoscopic and robotic. Since the introduction of Da Vinci robotic system in the early 2000s. It has been useful for more meticulous dissection of deep and narrow spaces and easier to gain access. In this article we describe our approach using the Da Vinci Xi robotic system in LPND and with a supplementary video.

Keywords: Lymph nodes, Robotic surgical procedures, Rectal neoplasms

Supplementary video file: This article contains supplementary material (https://doi.org/10.7602/ jmis.2020.23.2.103).

\author{
Received March 24, 2020 \\ Accepted June 4, 2020 \\ Corresponding author \\ In Kyu Lee \\ Division of Colorectal Surgery, \\ Department of Surgery, Seoul St. \\ Mary's Hospital, The Catholic \\ University of Korea College of \\ Medicine, 222 Banpo-daero, \\ Seocho-gu, Seoul 06591, Korea \\ Tel: $+82-2-2258-6104$ \\ Fax: +82-2-595-2822 \\ E-mail: cmcgslee@catholic.ac.kr \\ ORCID: \\ https://orcid.org/0000-0001-9074-5214
}

Copyright $\odot 2020$ The Journal of Minimally Invasive Surgery. All rights reserved.

This is an Open Access article distributed under the terms of the Creative Commons Attribution Non-Commercial License (http:// creativecommons.org/licenses/by-nc/4.0/) which permits unrestricted non-commercial use, distribution, and reproduction in any medium, provided the original work is properly cited.

\section{INTRODUCTION}

The current gold standard for patient with $\mathrm{T} 3$ or $\mathrm{T} 4$ with or without lymph node involvement is neoadjuvant chemoradiotherapy followed by total mesorectal excision (TME). ${ }^{1}$ There is a debate in the management of lateral pelvic lymph node (LPN) between western and eastern society. ${ }^{2-4}$ Western society consider LPN involvement as a distant metastasis and they treat it with chemoradiotherapy. ${ }^{2,3}$ On the other hand, eastern society consider LPN involvement as a local disease and they treat it with LPND. ${ }^{2-4}$ Different surgical approaches have been reported in the management of lateral pelvic lymph node dissection (LPND) including open, laparoscopic and robotic. ${ }^{5}$ In this article we will explain our method for LPND. This study was reviewed by the Institutional Review Board of Seoul St. Mary's Hospital and approved from exemption (IRB number: 2020-1040-0001).

\section{PROCEDURE}

Our technique for robotic low anterior or abdominal perineal resection with or without LPND is totally robotic and it have the same port placement. We use 'double docking surgery' Fig. 1. First phase is for inferior mesenteric vessel ligation and splenic mobilization (splenic phase), targeting the scope toward the descending colon with sigmoid junction. Af- 

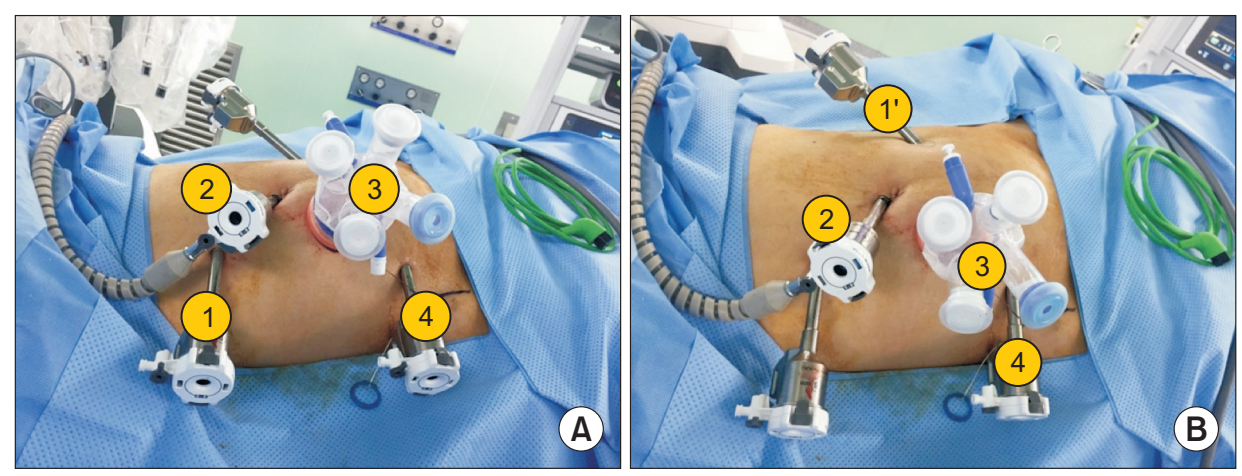

Fig. 1. Position of the trocars in splenic phase (A) and TME and LPLD phase (B).

ter the colonic mobilization, we begin TME phase and LPND (second phase) targeting the scope toward the rectum. We use four $8 \mathrm{~mm}$ port and one single port (glove port ${ }^{\oplus}$, Nelis). Distance among ports were at least $6 \mathrm{~cm}$ from each other to avoid collusion. The camera port (Arm 2) inserted at supra-umbilical on midline. After creating pneumoperitoneum, robotic camera is placed and checked the direction of spleen and the right anterior superior iliac spine (ASIS). A line drawn on the skin from the umbilical port toward the Right ASIS. Then at least $6 \mathrm{~cm}$ away from the umbilical port toward the right ASIS a transverse $4 \sim 4.5 \mathrm{~cm}$ incision is made for the glove port (Arm 3) and a $12 \mathrm{~mm}$ trocar with adjustable $8 \mathrm{~mm}$ cover is inserted (this port used also for specimen retrieval). This multi channeled port made suction (Levin tube Fig. 2) and rectal traction easier.

On the same line we measure at least $6 \mathrm{~cm}$ from port number 3 then go $2 \mathrm{~cm}$ toward the pubic tubercle and insert a 8 $\mathrm{mm}$ port (Arm 4). Next port (Arm 1 used for colon and splenic mobilization only) will be inserted in triangular fashion and at least $6 \mathrm{~cm}$ away from the umbilical and glove port. Last port (Arm 1' in TME and LPND) size $8 \mathrm{~mm}$ is inserted at the same umbilical port level toward the left side of the patient (this port used for TME and LPND).

After port insertion patient position in Trendelenburg position. A Cardiere grasper is used in Arm 1 and camera was fixed on arm 2. Stapling and monopolar or energy device were used through arm 3. Fenestrated grasper applied on arm 4. LPND usually start after the colon is completely resected with anvil insertion but before doing the anastomoses.

\section{OPERATIVE STEPS}

It can be simply divided to five steps:

First step is to dissect between Ureter and pelvic plexus. Secondly we identify the external iliac artery \& vein, and dissect towards the psoas muscle. Then we identify the common iliac artery bifurcation and dissect in between. After that we identify the obturator nerve and artery and dissect along them

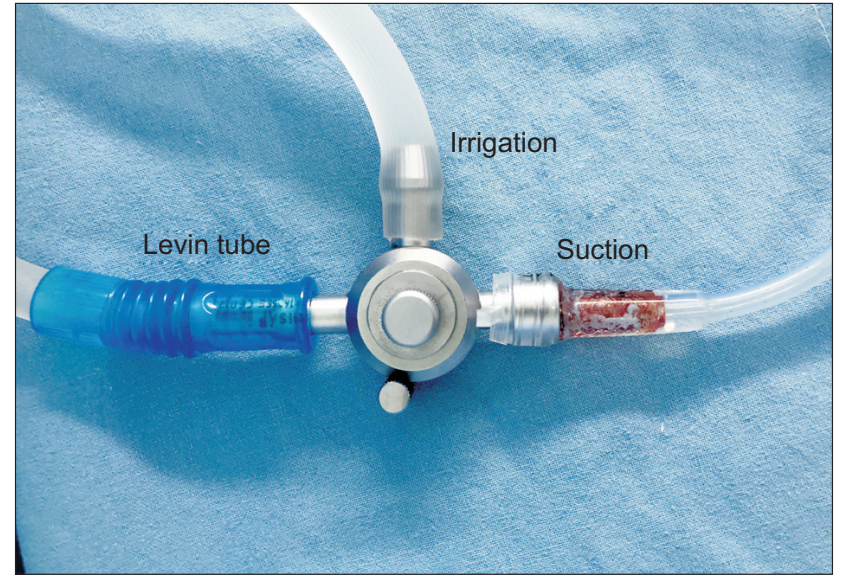

Fig. 2. Instrument for suction \& irrigation made by Levin tube.

and ligate the artery. The last step is to dissect the along the internal iliac artery distally towards the Alcock's canal (pudendal canal). After that the specimen is taken out as en bloc through the glove port in an endobag to avoid spillage of content.

\section{DISCUSSION}

Surgery is all about knowing the anatomy and exact planes. Now with the era of laparoscopic and robotic technology, surgeon can gain access to deep structure more easily with an excellent 3D view.

At the beginning of my practice for LPND I focused only for enlarged lymph node. After attending many cadaveric workshops and video presentations I started to know more and more about the anatomy of the lateral pelvic wall and better visualization of my dissection plan. Finally, I reached my goal for meticulous dissection and en bloc resection of the lymph nodes. Gentle traction and counter traction with meticulous dissection will yield a bloodless plan and decrease the incidence of devastating complication. During dissection knowing the major structure and dissection plan is an important step 
for complete and safe lymph node dissection.

Using the robotic system can give the surgeon the benefit of more steady dissection with an excellent articulation to gain access to the deep pelvic structure. Surgeon should be patient, attending courses and workshops to step up in their learning curve. There is no gold standard until now for the LPND and we are doing our best to standardize this technique for better patient safety and outcome.

\section{ORCID}

Suhail Abdullah Alturkistani, https://orcid.org/0000-0002-3086-6230

Alanoud Mohammed Alghanem, https://orcid.org/0000-0002-8083-3998

In Kyu Lee, https://orcid.org/0000-0001-9074-5214

\section{AUTHORS' CONTRIBUTIONS}

Conceptualization: In Kyu Lee. Formal analysis: In Kyu Lee. Methodology: Suhail Avdullah Alturkistani and In Kyu Lee. Writing-original draft: Suhail Avdullah Alturkistani and In Kyu Lee. Writing-review and editing: Suhail Avdullah Alturkistani, Alanoud Mohammed Alghanem, and In Kyu Lee.

\section{FUNDING}

None.

\section{ACKNOWLEDGMENTS}

None.

\section{REFERENCES}

1) Feeney G, Sehgal R, Sheehan M, et al. Neoadjuvant radiotherapy for rectal cancer management. World J Gastroenterol 2019;25: 4850-4869.

2) Moriya Y. Treatment of lateral pelvic nodes metastases from rectal cancer: the future prospective. G Chir 2013;34:245-248.

3) Yano H, Moran BJ. The incidence of lateral pelvic side-wall nodal involvement in low rectal cancer may be similar in Japan and the West. Br J Surg 2008;95:33-49.

4) Kobayashi H, Mochizuki H, Kato $\mathrm{T}$, et al. Outcomes of surgery alone for lower rectal cancer with and without pelvic sidewall dissection. Dis Colon Rectum 2009;52:567-576.

5) Bae SU, Saklani AP, Hur H, et al. Robotic and laparoscopic pelvic lymph node dissection for rectal cancer: short-term outcomes of 21 consecutive series. Ann Surg Treat Res 2014;86:76-82.

\section{CONFLICT OF INTEREST}

None. 